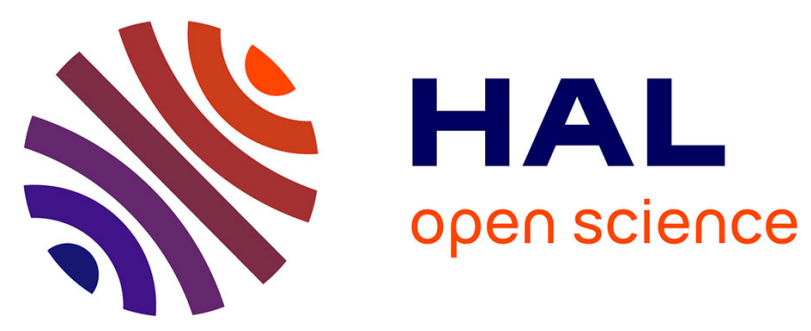

\title{
Design of a walking cyclic gait with single support phases and impacts for the locomotor system of a thirteen-link 3D biped using the parametric optimization
}

David Tlalolini, Yannick Aoustin, Christine Chevallereau

\section{- To cite this version:}

David Tlalolini, Yannick Aoustin, Christine Chevallereau. Design of a walking cyclic gait with single support phases and impacts for the locomotor system of a thirteen-link 3D biped using the parametric optimization. Multibody System Dynamics, 2010, 23 (1), pp.33-56. 10.1007/s11044-009-9175-1. hal-00794667

\section{HAL Id: hal-00794667 \\ https://hal.science/hal-00794667}

Submitted on 26 Feb 2013

HAL is a multi-disciplinary open access archive for the deposit and dissemination of scientific research documents, whether they are published or not. The documents may come from teaching and research institutions in France or abroad, or from public or private research centers.
L'archive ouverte pluridisciplinaire $\mathbf{H A L}$, est destinée au dépôt et à la diffusion de documents scientifiques de niveau recherche, publiés ou non, émanant des établissements d'enseignement et de recherche français ou étrangers, des laboratoires publics ou privés. 


\title{
Design of a walking cyclic gait with single support phases and impacts for the locomotor system of a thirteen-link 3D biped using the parametric optimization
}

\author{
David Tlalolini, \\ Yannick Aoustin* and Christine Chevallereau
}

the date of receipt and acceptance should be inserted later

\begin{abstract}
An algorithm of parametric optimization to achieve optimal cyclic gaits in space for a thirteen-link 3D bipedal robot with twelve actuated joints is proposed. The cyclic walking gait is composed of successive single support phases and impulsive impacts with full contact between the sole of the feet and the ground. The evolution of the joints are chosen as spline functions. The parameters to define the spline functions are determined using an optimization under constraints on the dynamic balance, on the ground reactions, on the validity of impact, on the torques and on the joints velocities. The cost functional considered is represented by the integral of the torques norm. The torques and the constraints are computed at sampling times during one step to evaluate the cost functional for a feasible walking gait. To improve the convergence of the optimization algorithm the explicit analytical gradient of the cost functional with respect to the optimization parameters is calculated using the recursive computation of torques. The algorithm is tested for a bipedal robot whose numerical walking results are presented.
\end{abstract}

\section{Keywords}

3D Bipedal robot, Robot dynamics, Fully actuated robot, Newton-Euler algorithm, Cyclic walking gait, Parametric optimization.

\section{Introduction}

The design of walking cyclic gaits for legged robots and particularly the bipeds has attracted the interest of many researchers for several decades. Due to the unilateral constraints of the biped with the ground and the great number of degrees of freedom, this problem is not trivial. Intuitive methods can be used to obtain walking gaits as in [1]. Using physical considerations, the authors of [1] defined polynomial functions in time for an experimental

David Tlalolini, Yannick Aoustin *(Corresponding author) and Christine Chevallereau are with IRCCyN, UMR 6597, École Centrale de Nantes, Université de Nantes, CNRS, France e-mail : David.TlaloliniRomero@irccyn.ec-nantes.fr, Yannick.Aoustin@irccyn.ec-nantes.fr, Christine.Chevallereau@irccyn.ecnantes.fr 
planar biped. This method is efficient. However, to build a bipedal robot and to choose the appropriate actuators or to improve the autonomy of a biped, an optimization algorithm can lead to very interesting results. In [2] the Pontryagin's principle is used to design impactless nominal trajectories for a planar biped with feet. However, the calculations are complex and difficult to extend to the 3D case. Furthermore the adjoint equations are not stable and highly sensitive to the initial conditions [3]. As a consequence a parametric optimization is a useful tool to find optimal motion. For example, in robotics, basis functions as polynomial functions, splines, truncated fourier series are used to approximate the motion of the joints, [4], [5], [6], [7], [8], [9] and [10]. The choice of optimization parameters is not unique. The torques, the Cartesian coordinates or joint coordinates can be used. Discrete values for the torques defined at sampling times are used as optimization parameters in [11]. However it is necessary, when the torque is an optimized variable, to use the direct dynamic model to find the joint accelerations. Then integrations are used to obtain the evolution of the reference trajectory in velocity and in position. Thus this approach requires much calculations: the direct dynamic model is complex and many evaluations of this model are used in the integration process. In [12], [13], [14], [15], [16] or [9] to overcome this difficulty, the parametric optimization defines the reference trajectories of Cartesian coordinates or joint ordinates for 2D bipeds with feet or without feet. An extension of this strategy is given in this paper to obtain a cyclic walking gait for a 3D biped with twelve motorized joints.

The evaluation of the cost criterion requires multibody system dynamics computations. The gradient of the criterion necessary in the optimization process is usually solved numerically through the finite difference method. This fact leads to an ill-conditioning and a poor convergence. Furthermore with finite difference approximations for the gradient, round-off errors appear. Then it is not possible to ensure a bounded condition number for the approximated Hessian. The optimization algorithms can stop prematurely [17]. Therefore some papers proposed optimization algorithms with the exact analytic gradient, [18], [8] and [9]. The problem of the optimal control and the recursive dynamics-based computer animation with the derivation of the explicit analytic gradients needed in the dynamic equations is addressed in [18]. The inverse dynamics model and the derivatives with respect to the path parameters, useful for the evaluation of the gradient and the Hessian are computed recursively for the general class of multi-body systems addressed in [8]. A walking gait with double support phases is designed for a five-link planar biped without actuated ankles in [9]. The calculation of the analytic gradient for the criterion and the constraints is made for this walking gait taking into account the characteristics of the over-actuated phases (double support), the under-actuated phases (single support) and the impacts.

A step (a half stride) of the cyclic walking gait is uniquely composed of a single support and an instantaneous double support which is modeled by passive impulsive equations. This walking gait is simpler than the human gait. But, with this simple model the coupling effect between the motion in frontal plane and sagittal plane can be studied. A finite time double support phase is not considered in this work currently because for rigid modeling of robot, a double support phase can usually be obtained only when the velocity of the swing leg tip before impact is null. This constraint has two effects. In the control process it will be difficult to touch the ground with a null velocity, as a consequence the real motion of the robot will be far from the ideal cycle. Furthermore, large torques are required to slow down the swing leg before the impact and to accelerate the swing leg at the beginning of the single support. The energy cost of such a motion is higher than a motion with impact in the case of a planar robot without feet [16], [9]. The evolution of joint variables are chosen as spline functions of time instead of usual polynomial functions to prevent oscillatory phenomenon during the optimization process (see [16], [19] or [20]). The coefficients of the spline func- 
tions are calculated as functions of initial, intermediate and final configurations, initial and final velocities of the robot. These configuration and velocity variables can be considered as optimization variables. Taking into account the impact and the fact that the desired walking gait is periodic, the number of optimization variables is reduced. In other study the periodicity conditions are treated as equality constraints [21]. The cost functional considered is the integral of the torque norm, which is a common criterion for the actuators of robotic manipulators, [4] and [22], [16] and [23]. During the optimization process, the constraints on the dynamic balance, on the ground reactions, on the validity of impact, on the limits of the torques, on the joints velocities and on the motion velocity of the bipedal robot are taken into account. Therefore an inverse dynamic model is calculated during the single phase to obtain the torques for a suitable number of sampling times. An impulsive model for the impact on the ground with complete surface of the foot sole of the swing leg is deduced from the dynamic model for the biped in double support phase. Then it is possible to evaluate he criterion cost, the constraints during the single support and at the impact.

The dynamic model for a 3D biped with twelve degrees of freedom is more complex than for a $2 \mathrm{D}$ biped with less degrees of freedom. So its computation cost is important in the optimization process and the use of Newton-Euler method to calculate the torque is more appropriate than the Lagrange method usually used. Then for the $3 \mathrm{D}$ biped, in single support, our model is founded on the Newton Euler algorithm, considering that the reference frame is connected to a stance foot. The walking study includes impact phase. The problem solved in [18] and [8] is to obtain an optimal motion beginning at a given state and ending at another given state. Furthermore authors used Lie theoretic formulation of the equations of motion and the analytic gradient. In our case the objective is to define cyclic walking for the 3D Biped. Lie theoretic formulation is avoided because for rigid bodies in serial or closed chains, recursive ordinary differential equations founded on the Newton-Euler algorithm is appropriate see [24]. Then explicit analytic gradient of the cost functional with respect to the parameters for the optimization problem is calculated using the recursive Newton-Euler algorithm to obtain the torques.

The paper is organized as follows. The 3D biped and its dynamic model are presented in Section 2. The cyclic walking gait and the constraints are defined in Section 3. The optimization parameters, optimization process and the cost functional are discussed in Section 4. The calculations to obtain the analytic gradient are detailed in Section 5. Furthermore a summary of the global optimization process is given in Section 5. Simulation results are presented in Section 6. Section 7 contains our conclusion and perspectives.

\section{Model of the bipedal robot}

\subsection{Biped model}

We considered an anthropomorphic bipedal robot with thirteen rigid links connected by twelve motorized joints to form a serial structure. It is composed of a torso, which is not directly actuated, and two identical open chains called legs which are connected at the hips. Each leg is composed of two massive links connected by a joint called knee. The link at the extremity of each leg is called foot which is connected at the leg by a joint called ankle. Each revolute joint is assumed to be independently actuated and ideal (frictionless). The ankles of the bipedal robot consist of the pitch and the roll axes, the knees consist of the pitch axis and the hips consist of the roll, pitch and yaw axes to constitute a biped walking system shown in figure 1 . The action to walk associates single support phases separated by impacts 
with full contact between the sole of the feet and the ground, thus a model in single support, and an impact model are derived. The dynamic model in single support is used to evaluate the required torque thus only the inverse dynamic model is necessary. The impact model is used to determine the velocity of the robot after the impact, the torques are zero during the impact, thus a direct impact model is required.

The periodic walk studied includes a symmetrical behavior when the support is on right leg and left leg, thus only the behavior during a step is computed, the behavior during the following step is deduced by symmetry rules. As a consequence only the modeling on right leg in support is considered in the following.

\subsection{Geometric description of the biped}

For a planar robot any parametrization of the robot can be used, for a 3D model of robot with many degrees of freedom a systematic parametrization of the robot must be developed. Many studies have been conducted for the robot manipulator, thus the parametrization proposed for the robot manipulator is re-used for the walking robot. The first difficulty is to choose a base link for a walking robot. Since the right leg is in support during all the studied step. The supporting foot is considered as base link.

To define the geometric structure of the biped we assume that the link 0 (stance foot) is the base of the bipedal robot while the link 12 (swing foot) is the terminal link. Therefore we have a simple open loop robot which the geometric structure can be described using the notation of Khalil and Kleinfinger [25]. The definition of the link frames is presented in figure 1 and the corresponding geometric parameters are given in table 1, where:

- $a(j)$ denotes the frame antecedent to the frame $j$

- The geometric parameters $\left(\alpha_{j}, \theta_{j}, r_{j}, d_{j}\right)$ determine the location of the frame $j$ with respect to its antecedent $a(j)$.

The frame $R_{0}$, which is fixed to the tip of the right foot (determined by the width $l_{p}$ and the length $L_{p}$ ), is defined such that the axis $z_{0}$ is directed along the axis of frontal joint ankle. The frame $R_{13}$ is fixed to the tip of the left foot with the same orientation as $R_{0}$.

\begin{tabular}{|c|c|c|c|c|c|}
\hline$j$ & $a(j)$ & $\alpha_{j}$ & $\theta_{j}$ & $r_{j}$ & $d_{j}$ \\
\hline 1 & 0 & 0 & $q_{1}$ & $l_{1}$ & $d_{1}$ \\
2 & 1 & $\frac{\pi}{2}$ & $q_{2}$ & 0 & 0 \\
3 & 2 & 0 & $q_{3}$ & 0 & $d_{3}$ \\
4 & 3 & 0 & $q_{4}$ & $l_{4}$ & $d_{4}$ \\
5 & 4 & $-\frac{\pi}{2}$ & $q_{5}-\frac{\pi}{2}$ & 0 & 0 \\
6 & 5 & $-\frac{\pi}{2}$ & $q_{6}$ & 0 & 0 \\
7 & 6 & 0 & $q_{7}$ & 0 & $d_{7}$ \\
8 & 7 & $\frac{\pi}{2}$ & $q_{8}-\frac{\pi}{2}$ & 0 & 0 \\
9 & 8 & $-\frac{\pi}{2}$ & $q_{9}$ & 0 & 0 \\
10 & 9 & 0 & $q_{10}$ & $l_{10}=l_{4}$ & $d_{10}=d_{4}$ \\
11 & 10 & 0 & $q_{11}$ & 0 & $d_{11}=d_{3}$ \\
12 & 11 & $\frac{\pi}{2}$ & $q_{12}$ & 0 & 0 \\
13 & 12 & 0 & $q_{13}$ & $l_{13}=-l_{1}$ & $d_{13}=d_{1}$ \\
\hline
\end{tabular}

Table 1: Geometric parameters of the biped. 


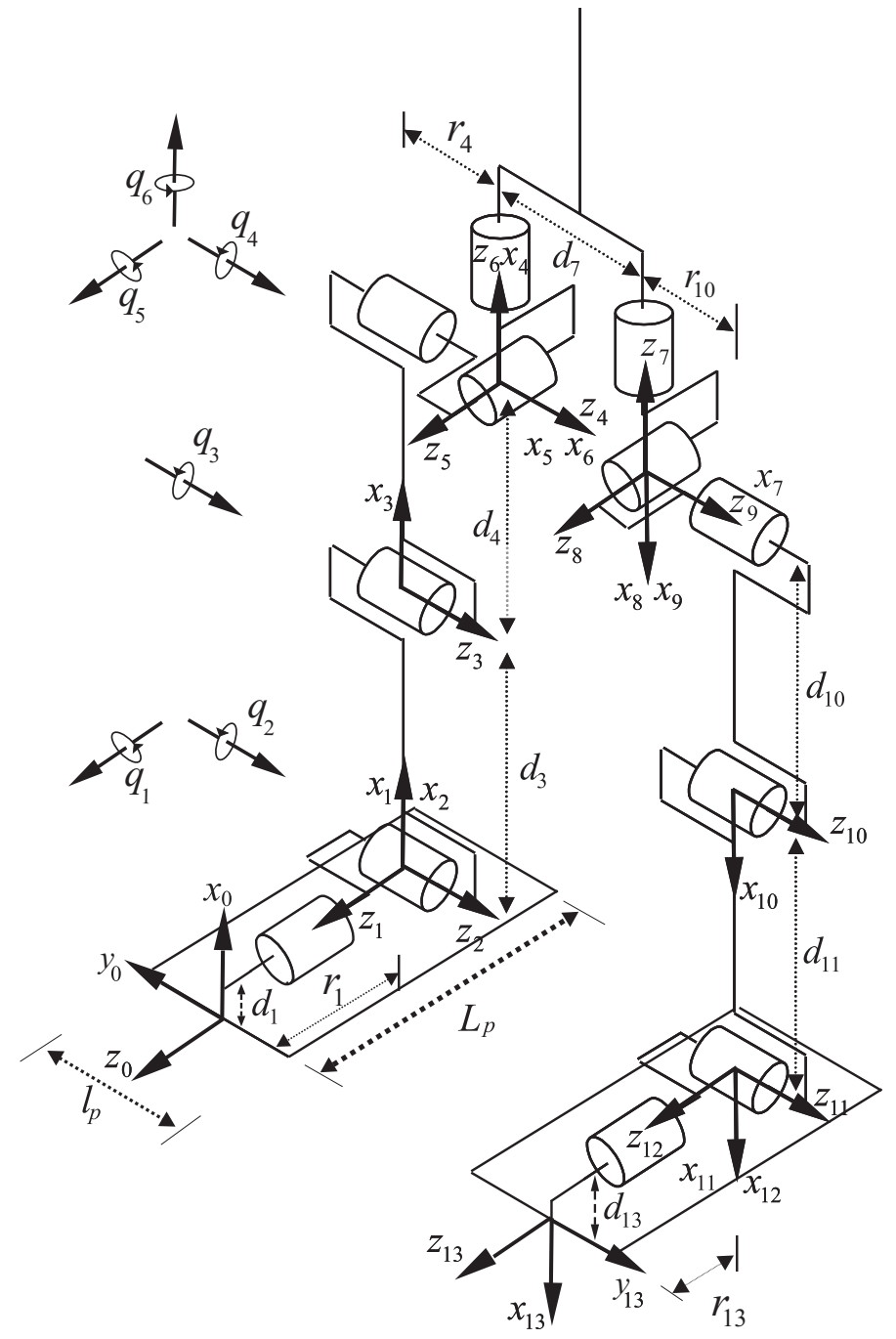

Fig. 1: The multi-body model and link frames of the bipedal robot.

2.3 Dynamic model in single support phase

During the single support phase, our objective is only to determine the inverse dynamic model. The joint position, velocity and acceleration are known. The actuator torques must be calculated. Since the contact between the stance foot and the ground is unilateral, the ground reaction (forces and torques) must also be deduced. The Newton-Euler algorithm (see [26]) can be adapted to determine the ground wrench.

During the single support phase the stance foot is assumed to remain in flat contact on the ground, i.e., no sliding motion, no take-off, no rotation. Therefore the biped is equivalent to a 12-DoF manipulator. Let $\mathbf{q} \in \mathbb{R}^{12}$ be the generalized coordinates, where $q_{1}, \ldots, q_{12}$ denote the relative angles of the joints, $\dot{\mathbf{q}} \in \mathbb{R}^{12}$ and $\ddot{\mathbf{q}} \in \mathbb{R}^{12}$ are the velocity vector and the acceleration vector respectively. The dynamic model is represented by the following relation 


$$
\left[\begin{array}{c}
\mathbf{R}_{0} \\
\Gamma
\end{array}\right]=N E\left(\mathbf{q}, \dot{\mathbf{q}}, \ddot{\mathbf{q}}, \mathbf{R}_{13}\right)
$$

where $\Gamma \in \mathbb{R}^{12}$ is the joint torques vector, $\mathbf{R}_{0} \in \mathbb{R}^{6}$ is the ground wrench (forces and moments) exerted by the ground on the stance foot and $\mathbf{R}_{13} \in \mathbb{R}^{6}$ represents the wrench, exerted by the terminal link on the ground. In single support phase $\mathbf{R}_{13}=\mathbf{0}_{6 \times 1}$.

\subsubsection{Newton-Euler algorithm}

The Newton-Euler method permits to calculate the dynamic model as defined in equation (1). This method proposed by Luh, Walker et Paul [27] is based on two recursive calculations. Associated with our choice of parametrization the following algorithm is obtained [26]. The forward calculation, from the base (stance foot) to the terminal link (swing foot) determines the velocity, the accelerations and the total forces and moments for each link. Then the backward calculations, from swing foot to stance foot, gives the joint torques and reaction forces using equation of equilibrium of each link successively.

\section{Forward recursive equations}

Taking into account that the bipedal robot remains flat on the ground, the initial conditions are

$$
{ }^{0} \omega_{0}=\mathbf{0}_{6 \times 1},{ }^{0} \dot{\omega}_{0}=\mathbf{0}_{6 \times 1} \text { and }{ }^{0} \dot{\mathbf{V}}_{0}=-\left[\begin{array}{lll}
g & 0 & 0
\end{array}\right]^{t}
$$

the real acceleration is ${ }^{0} \dot{\mathbf{V}}_{0}=\mathbf{0}_{6 \times 1}$ but, the choice to write ${ }^{0} \dot{\mathbf{V}}_{0}=-\left[\begin{array}{lll}g & 0 & 0\end{array}\right]^{t}$, where $g$ is the gravity acceleration, allows to take into account the gravity effect.

For the link $j$ with its associated frame $R_{j}$, and considering the link $j-1$ as its antecedent, its angular velocity ${ }^{j} \omega_{j}$, and the linear velocity ${ }^{j} \mathbf{V}_{j}$ of the origin $O_{j}$ of $R_{j}$ are

$$
\begin{aligned}
{ }^{j} \omega_{j} & ={ }^{j} \omega_{j-1}+\bar{\sigma}_{j} \dot{\mathbf{q}}_{j}{ }^{j} \mathbf{a}_{j} \\
{ }^{j} \mathbf{V}_{j} & ={ }^{j} \mathbf{A}_{j-1}\left({ }^{j-1} \mathbf{V}_{j-1}+{ }^{j-1} \stackrel{\wedge}{\omega_{j-1}^{j-1}} \mathbf{P}_{j}\right)+\sigma_{j} \dot{\mathbf{q}}_{j}{ }^{j} \mathbf{a}_{j}
\end{aligned}
$$

with ${ }^{j} \mathbf{A}_{j-1}$, the orientation matrix of the frame $R_{j-1}$ in the frame $R_{j}, \sigma_{j}=0$ when the joint $j$ is a revolute joint, $\sigma_{j}=1$ when the joint $j$ is prismatic joint and $\bar{\sigma}_{j}=1-\sigma_{j},{ }^{j} \mathbf{a}_{j}$ is an unit vector along the $z_{j}$ axis, ${ }^{j-1} \mathbf{P}_{j}$ is the vector expressing the origin of frame $R_{j}$ in the frame $R_{j-1}$. The angular acceleration of link $j$ and the linear acceleration of the origin $O_{j}$ of $R_{j}$ are

$$
\begin{aligned}
{ }^{j} \dot{\boldsymbol{\omega}}_{j} & ={ }^{j} \mathbf{A}_{j-1}^{j-1} \dot{\boldsymbol{\omega}}_{j-1}+\overline{\boldsymbol{\sigma}}\left(\ddot{\mathbf{q}}_{j}{ }^{j} \mathbf{a}_{j}+{ }^{j} \hat{\omega}_{j-1} \dot{\mathbf{q}}_{j}^{j} \mathbf{a}_{j}\right) \\
{ }^{j} \dot{\mathbf{V}}_{j} & ={ }^{j} \mathbf{A}_{j-1}\left({ }^{j-1} \dot{\mathbf{V}}_{j-1}+{ }^{j-1} \mathbf{U}_{j-1}{ }^{j-1} \mathbf{P}_{j}\right)+\sigma_{j}\left(\ddot{\mathbf{q}}_{j}{ }^{j} \mathbf{a}_{j}+2{ }^{j} \hat{\omega}_{j-1} \dot{\mathbf{q}}_{j}^{j} \mathbf{a}_{j}\right)
\end{aligned}
$$

where ${ }^{j} \mathbf{U}_{j}={ }^{j} \dot{\boldsymbol{\omega}}_{j}+{ }^{j} \hat{\omega}_{j}{ }^{j} \hat{\omega}_{j}$.

The total inertial forces and moments for the link $j$ are

$$
\begin{aligned}
{ }^{j} \mathbf{F}_{j} & =M_{j}{ }^{j} \dot{\mathbf{V}}_{j}+{ }^{j} \mathbf{U}_{j}{ }^{j} \mathbf{M} \mathbf{S}_{j} \\
{ }^{j} \mathbf{M}_{j} & ={ }^{j} \mathbf{J}_{j}{ }^{j} \dot{\boldsymbol{\omega}}_{j}+{ }^{j} \hat{\omega}_{j}\left({ }^{j} \mathbf{J}_{j}{ }^{j} \boldsymbol{\omega}_{j}\right)+{ }^{j} \hat{\mathbf{M S}}{ }_{j}^{j} \dot{\mathbf{V}}_{j}
\end{aligned}
$$

with ${ }^{j} \mathbf{J}_{j}$ inertia tensor of the link $j$ with respect to the frame $R_{j},{ }^{j} \mathbf{M S} \mathbf{S}_{j}$ is the first moments vector of the link $j$ around the origin of the frame $R_{j}$ and $M_{j}$ is the mass of the link $j$. The 
antecedent link to the link 0 (stance foot) is not defined. For the iteration of the stance foot, only the equations (7) and (8) are used.

\section{Backward recursive equations}

The backward recursive equations are given as, for $j=12, \ldots, 0$

$$
\begin{aligned}
{ }^{j} \mathbf{f}_{j} & ={ }^{j} \mathbf{F}_{j}+{ }^{j} \mathbf{f}_{j+1} \\
{ }^{j-1} \mathbf{f}_{j} & ={ }^{j-1} \mathbf{A}_{j}{ }^{j} \mathbf{f}_{j} \\
{ }^{j} \mathbf{m}_{j} & ={ }^{j} \mathbf{M}_{j}+{ }^{j} \mathbf{A}_{j+1}{ }^{j+1} \mathbf{m}_{j+1}+{ }^{j} \hat{\mathbf{P}}_{j+1}{ }^{j} \mathbf{f}_{j+1}
\end{aligned}
$$

where ${ }^{j} \mathbf{f}_{j}$ is the resultant force, exerted on the link $j$ by its antecedent and by the actuators $j,{ }^{j} \mathbf{m}_{j}$ is the resultant moment, exerted on link $j$ by its antecedent and by the actuator $j$. These recursive equations will be initialized by the forces and moments exerted on the terminal link by the environment ${ }^{n} \mathbf{f}_{n+1}$ and ${ }^{n} \mathbf{m}_{n+1}$. In single support ${ }^{n} \mathbf{f}_{n+1}={ }^{12} \mathbf{f}_{13}=\mathbf{0}_{3 \times 1}$, ${ }^{n} \mathbf{m}_{n+1}={ }^{12} \mathbf{m}_{13}=\mathbf{0}_{3 \times 1}$, with $\mathbf{R}_{13}=\left[{ }^{12} \mathbf{f}_{13},{ }^{12} \mathbf{m}_{13}\right]^{t}$. When $j=0, \mathbf{R}_{0}=\left[{ }^{0} \mathbf{f}_{0},{ }^{0} \mathbf{m}_{0}\right]^{t}$ represents the ground reaction force and moment expressed in the frame $R_{0}$.

If we neglect the friction and the motor inertia effects, the torque (or the force) $\Gamma_{j}$, is obtained by projecting $\mathbf{m}_{j}$ (or $\mathbf{f}_{j}$ ) along the joint axis $\left(z_{j}\right)$

$$
\Gamma_{j}=\left(\sigma_{j}{ }^{j} \mathbf{f}_{j}+\bar{\sigma}_{j}{ }^{j} \mathbf{m}_{j}\right)^{t}{ }^{j} \mathbf{a}_{j}
$$

$\Gamma_{0}$ is not defined, since there is no actuator.

\subsubsection{The zero Moment Point (ZMP) position}

The ground reaction wrench is known in the frame $R_{0}$. This frame is associated with the stance foot, and the axis $y_{0}, z_{0}$ defined the sole of the stance foot. The position of the ZMP which is the point of the sole such that the moment exerted by the ground is zero along the axis $y_{0}$ and $z_{0}$ is such that:

$$
\begin{aligned}
& y_{Z M P}=\frac{-{ }^{0} m_{0 z}}{{ }^{0} f_{0 x}} \\
& z_{Z M P}=\frac{{ }^{0} m_{0 y}}{{ }^{0} f_{0 x}}
\end{aligned}
$$

If the position of $Z M P$ is within the support polygon, the biped robot is in dynamic equilibrium, the stance foot remains flat on the ground.

\subsection{Impact model for the instantaneous double support}

At the impact, the previous supporting foot becomes the swing foot, and its velocity after impact can be different from zero. As a consequence the parametrization of the biped must be able to describe a non fixed stance foot. Since the dynamic model is calculated with the Newton-Euler algorithm, it is very convenient to define the velocity of the link 0 with the Newton variables: $\mathbf{V}_{0}$ the linear velocity of the origin of the frame $R_{0}$ and $\omega_{0}$ the angular velocity of the frame $R_{0}$. For the impact model, or the double support model the biped's position is expressed by $\mathbf{X}=\left[\mathbf{X}_{0}, \alpha_{0}, \mathbf{q}\right]^{t} \in \mathbb{R}^{18}, \mathbf{X}_{0}$ and $\alpha_{0}$ are the position and the orientation variables of the frame $R_{0}$; the robot velocity is $\mathbf{V}=\left[{ }^{0} \mathbf{V}_{0},{ }^{0} \omega_{0}, \dot{\mathbf{q}}\right]^{t} \in \mathbb{R}^{18}$ and the robot acceleration is $\dot{\mathbf{V}}=\left[{ }^{0} \dot{\mathbf{V}}_{0},{ }^{0} \dot{\omega}_{0}, \ddot{\mathbf{q}}\right]^{t} \in \mathbb{R}^{18}$. 


\subsubsection{Dynamic model in double support}

The impact model is deduced from the dynamic model in double support, when we assume that the acceleration of the robot and the reaction force are Dirac delta-functions.

The dynamical model in double support can be written:

$$
\mathbf{D}(\mathbf{X}) \dot{\mathbf{V}}+\mathbf{C}(\mathbf{V}, \mathbf{q})+\mathbf{G}(\mathbf{X})+\mathbf{D}_{12} \mathbf{R}_{13}=\mathbf{D}_{\Gamma} \Gamma+\mathbf{D}_{0} \mathbf{R}_{0}
$$

where $\mathbf{D} \in \mathbb{R}^{18 \times 18}$ is the symmetric definite positive inertia matrix, $\mathbf{C} \in \mathbb{R}^{18}$ represents the Coriolis and centrifugal forces, $\mathbf{G} \in \mathbb{R}^{18}$ is the vector of gravity. $\mathbf{R}_{0}=\left[{ }^{0} \mathbf{f}_{0},{ }^{0} \mathbf{m}_{0}\right]^{t}$ is the vector of the ground reaction forces on the stance foot, $\mathbf{R}_{13}=\left[{ }^{12} \mathbf{f}_{13},{ }^{12} \mathbf{m}_{13}\right]$ represents the vector of forces exerted by the swing foot on the ground, $\mathbf{D}_{12}, \mathbf{D}_{\Gamma}$ and $\mathbf{D}_{0}$ are matrices that allows to take into account the forces and torques in the dynamic model.

The model of impact can be deduced from (14) and is:

$$
\mathbf{D}(\mathbf{X}) \Delta \mathbf{V}+\mathbf{D}_{12} \mathbf{I}_{13}=\mathbf{D}_{0} \mathbf{I}_{0}
$$

where $\mathbf{I}_{13}$ and $\mathbf{I}_{0}$ are the intensity of Dirac delta-function for the forces $\mathbf{R}_{13}$ and $\mathbf{R}_{0} . \Delta \mathbf{V}$ is the variation of velocity at the impact, $\Delta \mathbf{V}=\mathbf{V}^{+}-\mathbf{V}^{-}$, where $\mathbf{V}^{-}$is the velocity of the robot before impact and $\mathbf{V}^{+}$its velocity after impact.

The impact is assumed to be inelastic with complete surface of the foot sole touching the ground. This means that the velocity of the swing foot impacting the ground is zero after impact. Two cases are possible after an impact: the right foot (previous stance foot) takes off the ground or both feet remain on the ground. In the first case, the vertical component of the velocity of the taking-off foot just after an impact must be directed upwards and the impulsive ground reaction in this foot equals zeros $\mathbf{I}_{0}=\mathbf{0}_{6 \times 1}$. In the second case, the right foot velocity has to be zero just after an impact. The ground produces impulsive forces in both feet. This implies that the vertical component of the impulsive ground reaction are directed upwards. An impacting foot with zero velocity at impact, is a solution of the two cases, there is no impact, the reaction forces on the two legs are null and the velocities of the two feet after impact are null.

For our numerical tests, for the studied bipedal robot, only the first case gives a valid solution. The swing foot velocity is zero before the impact (and there is no impact) or the previous stance foot does not remain on the ground after the impact. Thus, the impact dynamic model is (see [28] and [29])

$$
\begin{aligned}
\mathbf{D}(\mathbf{X}) \Delta \mathbf{V} & =-\mathbf{D}_{12} \mathbf{I}_{13} \\
\mathbf{D}_{12}^{t} \mathbf{V}^{+} & =\mathbf{0}_{6 \times 1} \\
{\left[\begin{array}{l}
{ }^{0} \mathbf{V}_{0}^{-} \\
{ }^{0} \omega_{0}^{-}
\end{array}\right] } & =\left[\begin{array}{l}
\mathbf{0}_{3 \times 1} \\
\mathbf{0}_{3 \times 1}
\end{array}\right]
\end{aligned}
$$

These equations form a system of linear equations which determines the impulse forces $\mathbf{I}_{13}$ and the velocity vector of the biped after impact $\mathbf{V}^{+}$.

$$
\begin{aligned}
\mathbf{I}_{13} & =\left(\mathbf{D}_{12}^{t} \mathbf{D}^{-1} \mathbf{D}_{12}\right)^{-1} \mathbf{D}_{12}^{t} \mathbf{V}^{-} \\
\mathbf{V}^{+} & =-\mathbf{D}^{-1} \mathbf{D}_{12}\left(\mathbf{D}_{12}^{t} \mathbf{D}^{-1} \mathbf{D}_{12}\right)^{-1} \mathbf{D}_{12}^{t} \mathbf{V}^{-}+\mathbf{V}^{-}
\end{aligned}
$$


2.4.2 Calculation of the matrix $\mathbf{D}_{12}$

The wrench $\mathbf{R}_{13}=\left[{ }^{12} \mathbf{f}_{13},{ }^{12} \mathbf{m}_{13}\right]$ is naturally expressed in the frame $R_{12}$. The velocities of link 12 with respect to the biped velocity $\mathbf{V}$, can be expressed as

$$
\left[\begin{array}{c}
\mathbf{V}_{12} \\
\omega_{12}
\end{array}\right]=\left[\begin{array}{c}
\mathbf{V}_{0}+\hat{\omega}_{0}^{0} \mathbf{P}_{12} \\
\omega_{12}
\end{array}\right]+\mathbf{J}_{12} \dot{\mathbf{q}}
$$

where ${ }^{0} \mathbf{P}_{12}$ is the vector linking the origin of the frame $R_{0}$ and the origin of the frame $R_{12}$ expressed in the frame $R_{0}, \mathbf{J}_{12} \in \mathbb{R}^{6 \times 12}$ is the Jacobian matrix of the robot, $\mathbf{J}_{12} \dot{\mathbf{q}}$ represents the effect of the joint velocities on the Cartesian velocity of link 12. The velocities $\mathbf{V}_{12}$ and $\omega_{12}$ must be expressed in the frame $R_{12}$, thus we write:

$$
\left[\begin{array}{c}
{ }^{12} \mathbf{V}_{12} \\
{ }^{12} \omega_{12}
\end{array}\right]=\left[\begin{array}{cc}
{ }^{12} \mathbf{A}_{0}-{ }^{12} \mathbf{A}_{0}{ }^{0} \hat{\mathbf{P}}_{12} \\
\mathbf{0}_{3 \times 3} & { }^{12} \mathbf{A}_{0}
\end{array}\right]\left[\begin{array}{c}
{ }^{0} \mathbf{V}_{0} \\
{ }^{0} \omega_{0}
\end{array}\right]+{ }^{12} \mathbf{J}_{12} \dot{\mathbf{q}}
$$

where ${ }^{12} \mathbf{A}_{0} \in \mathbb{R}^{3 \times 3}$ is the rotation matrix, which defines the orientation of the frame $R_{0}$ with respect to the frame $R_{12}$. The expression (22) can be represented in matrix form as

$$
\left[\begin{array}{c}
{ }^{12} \mathbf{V}_{12} \\
{ }^{12} \omega_{12}
\end{array}\right]=\left[\begin{array}{cc|c}
{ }^{12} \mathbf{A}_{0} & -{ }^{12} \mathbf{A}_{0}{ }^{0} \hat{\mathbf{P}}_{12} & { }^{12} \mathbf{J}_{12}
\end{array}\right] \mathbf{V} .
$$

Thus $\mathbf{D}_{12}$ has the following structure

$$
\mathbf{D}_{12}=\left[\begin{array}{cc|c}
{ }^{12} \mathbf{A}_{0} & -{ }^{12} \mathbf{A}_{0}{ }^{0} \hat{\mathbf{P}}_{12} & { }^{12} \mathbf{J}_{12} \\
\mathbf{0}_{3 \times 3} & { }^{12} \mathbf{A}_{0} & { }^{t}
\end{array}\right.
$$

\subsubsection{Calculation of the inertia matrix $\mathbf{D}$}

Following the same way, as the wrench $\mathbf{R}_{0}$ is applied on the stance leg, in equation (14), $\mathbf{D}_{0}=\left[\mathbf{I}_{6 \times 6} \mid \mathbf{0}_{12 \times 6}\right]^{t} \in \mathbb{R}^{18 \times 6}$. The matrix $\mathbf{D}_{\Gamma}$ defines the actuated joint thus we have : $\mathbf{D}_{\Gamma}=$ $\left[\mathbf{0}_{6 \times 12} \mid \mathbf{I}_{12 \times 12}\right]^{t} \in \mathbb{R}^{18 \times 12}$.

When no force is applied on the swing leg, the dynamic model (14) becomes:

$$
\mathbf{D}(\mathbf{X}) \dot{\mathbf{V}}+\mathbf{C}(\mathbf{V}, \mathbf{q})+\mathbf{G}(\mathbf{X})=\left[\begin{array}{c}
\mathbf{R}_{0} \\
\Gamma
\end{array}\right]
$$

Since the stance foot is assumed to remain in flat contact, the resultant ground reaction force/moment $\mathbf{R}_{0}$ and the torques $\Gamma$ can be computed using the Newton-Euler algorithm (see section 2.3). According to the method of Walker [30], the matrix $\mathbf{D}$ is calculated by the algorithm of Newton-Euler, by noting from (14), that the $i^{\text {th }}$ column of $\mathbf{D}$ is equal to $\left[\begin{array}{c}\mathbf{R}_{0} \\ \Gamma\end{array}\right]$ if

$$
\mathbf{V}=\mathbf{0}_{18 \times 1}, g=0, \dot{\mathbf{V}}=\mathbf{e}_{i}, \mathbf{R}_{13}=\mathbf{0}_{6 \times 1}
$$

$\mathbf{e}_{i} \in \mathbb{R}^{18 \times 1}$ is the unit vector, whose elements are zero except the $i^{\text {th }}$ element which is equal to 1 . The vectors $\mathbf{C}(\mathbf{V}, \mathbf{q})$ and $\mathbf{G}(\mathbf{X})$ can be obtained in the same way that $\mathbf{D}$, however, for the impact model the knowledge of these vectors are not necessary. 


\section{Definition of the walking cycle}

Because a walking biped gait is a periodical phenomenon our objective is to design a cyclic biped gait. A complete walking cycle is composed of two phases: a single support phase and a double support phase which is modeled through passive impact equations. The single support phase begins with one foot which stays on the ground while the other foot swings from the rear to the front. We assume that the double support phase is instantaneous. This means that when the swing leg touches the ground the stance leg takes off. There are two facets to be considered for this problem. The definition of reference trajectories and the method to determine a particular solution of it. This section is devoted to the definition of reference trajectories. The optimal process to choose the best solution of parameters, allowing a symmetric step from the point of view of a given cost functional will be described in the next section.

\subsection{Cyclic walking trajectory}

Since the initial configuration is a double support configuration, both feet are on the ground, the twelve joint coordinates are not independent. Because the absolute frame is attached to the right foot we define the situation of the left foot by $\left(y_{l f}, z_{l f}\right)$ and the situation of the middle of the hips $\left(x_{h}, y_{h}, z_{h}, \theta_{h}\right)$, both expressed in $R_{0}$ frame. $\left(y_{l f}, z_{l f}\right)$ are the Cartesian coordinates, in the horizontal plane, of the left foot position, $\left(x_{h}, y_{h}, z_{h}\right)$ is the hip position and $\theta_{h}$ defines the hip pitching angle. The two others parameters, orientation for the middle efthe hips in frontal and transverse planes, are chosen to be equal to zero. The orientation of left foot is also chosen to be equal to zero. The values of the joint variables are solution of the inverse kinematics problem for a leg, which may also be considered as a 6-link manipulator. The problem is solved with a symbolic software, (SYMORO+, see [25]).

Let us consider, for the cyclic walking gait, the current step in the time interval $[0, T]$. In order to deduce the final configuration of the bipedal robot at time $t=T$, we impose a symmetric role of the two legs, therefore from the initial configuration $\mathbf{q}_{0}=\mathbf{q}(t=0)$ in double support, the final configuration $\mathbf{q}_{T}=\mathbf{q}(t=T)$ in double support is deduced as:

$$
\mathbf{q}_{T}=\mathbf{E} \mathbf{q}_{0}
$$

where $\mathbf{E} \in \mathbb{R}^{12 \times 12}$ is an inverted diagonal matrix which describes the exchange of legs.

Taking into account the impulsive impact (16)-(18), we can compute the velocity vector of the biped after the impact. Therefore, the joint rates after impact, $\dot{\mathbf{q}}^{+}$, can be calculated when the joint velocities before the impact, $\dot{\mathbf{q}}^{-}$, is known. The use of the defined matrix $\mathbf{E}$ allows us to calculate the initial joint rates $\dot{\mathbf{q}}_{0}=\dot{\mathbf{q}}(t=0)$ for the current step as:

$$
\dot{\mathbf{q}}_{0}=\mathbf{E} \dot{\mathbf{q}}^{+} .
$$

By this way the conditions of cyclic motion are satisfied and the initial and final velocities and the configuration are completly defined by $q_{0}$ and $\dot{q}^{-}$.

\subsection{Constraints}

In order to insure that the trajectory is possible, many constraints have to be considered. 
3.2.1 Magnitude constraints on position, velocities and torque

- Each actuator has physical limits such that

$$
\left|\Gamma_{i}\right|-\Gamma_{i, \max } \leq 0, \text { for } i=1, \ldots, 12
$$

where $\Gamma_{i, \max }$ denotes the maximum value for each actuator.

$$
\left|\dot{q}_{i}\right|-\dot{q}_{i, \max } \leq 0, \quad \text { for } i=1, \ldots, 12
$$

where $\dot{q}_{i, \max }$ denotes the maximum joint rate for each actuator.

- The upper and lower bounds of joints for the configurations during the motion are:

$$
q_{i, \min } \leq q_{i} \leq q_{i, \max }, \quad \text { for } i=1, \ldots, 12
$$

$q_{i, \min }$ and $q_{i, \max }$ respectively stands for the minimum and maximum joint limits.

\subsubsection{Geometric constraints in double support phase}

- The distance $d$ (hip, foot) between the foot in contact with the ground and the hip must remain within a maximal value, i.e.,

$$
d(\text { hip, foot }) \leq l_{\text {hip }}
$$

This condition must hold for final configurations of the double support phase.

- In order to avoid the internal collision of both feet through the lateral axis the heel and the toe of the left foot must satisfy

$$
y_{\text {heel }} \leq-a \text { and } y_{\text {toe }} \leq-a
$$

with $a>\frac{l_{p}}{2}$ and $l_{p}$ is the width of right foot.

\subsubsection{Walking constraints}

- During the single support phase to avoid collisions of the swing leg with the stance leg or with the ground, constraints on the positions of the four corners of the swing foot are defined.

- We must take into account the constraints on the ground reaction $\mathbf{R}_{0}=\left[R_{0 x}, R_{0 y}, R_{0 z}\right]^{t}$ for the stance foot in single support phase as well as impulsive forces $\mathbf{I}_{13}=\left[I_{13 x}, I_{13 y}, I_{13 z}\right]^{t}$ on the foot touching the ground in instantaneous double support phase. The ground reaction forces in single support and the impulsive forces at the impact must be directed upward, then the conditions of no take off are deduced:

$$
\begin{aligned}
& R_{0 x} \geq 0 \\
& I_{13 x} \geq 0 .
\end{aligned}
$$

The ground reaction in single support and the impulsive forces at the impact must be inside a friction cone defined by the friction coefficient $\mu$. This is equivalent to write

$$
\begin{aligned}
\sqrt{R_{0 y}^{2}+R_{0 z}^{2}} & \leq \mu R_{0 x} \\
\sqrt{I_{13 y}^{2}+I_{13 z}^{2}} & \leq \mu I_{13 x}
\end{aligned}
$$


- In order to maintain the balance in dynamic walking, the Zero Moment Point which is equivalent to the Center of Pressure $(C o P)$, (see [31], [32], [33]), must be within the interior of the support polygon of the biped stance foot. Then for a rectangular foot the CoP must satisfy

$$
\begin{gathered}
\frac{-l_{p}}{2}<\mathrm{CoP}_{y}<\frac{l_{p}}{2}, \\
-L_{p}<\operatorname{CoP}_{z}<0
\end{gathered}
$$

where $l_{p}$ is the width and $L_{p}$ is the length of the feet.

- An average walking rate is imposed. Thus

$$
V^{d} T-d=0
$$

where $d$ is the step length, $T$ is the step duration and $V^{d}$ is a desired speed of walking.

\section{Parametric optimization}

\subsection{The cubic spline}

The biped is driven by 12 torques, and its configuration is given in single support phase by 12 coordinates grouped in vector $\mathbf{q}$. To define the joint evolution, cubic spline functions [34] are used for constructing the joint trajectories. For each joint $j,(j=1, \ldots, 12)$ a cubic spline function has the form:

$$
q_{j}(t)=\left\{\begin{array}{ccc}
\varphi_{j, 1}(t) & \text { if } & t_{1} \leq t<t_{2} \\
\varphi_{j, 2}(t) & \text { if } & t_{2} \leq t<t_{3} \\
\vdots & & \vdots \\
\varphi_{j, n-1}(t) & \text { if } & t_{n-1} \leq t \leq t_{n}
\end{array}\right.
$$

where $n$ is the number of selected knots. $\varphi_{j 1}(t), \ldots, \varphi_{j n-1}(t)$ are polynomials of third-order such that:

$$
\varphi_{j, k}(t)=\sum_{i=0}^{3} a_{j, k}^{i}\left(t-t_{k}\right)^{i}, \text { for } t \in\left[t_{k}, t_{k+1}\right], k=1, \ldots, n-1
$$

where $a_{j, k}^{i}$ are calculated such that the position, velocity and acceleration are always continuous in $t_{1}, \ldots, t_{n}$. The cubic spline functions are uniquely defined by specifying an initial configuration $\mathbf{q}_{0}$, an initial velocity $\dot{\mathbf{q}}_{0}$ (both at $t=t_{1}=0$ ), a final configuration $\mathbf{q}_{T}$, and a final velocity $\dot{\mathbf{q}}_{T}$ (both at $t=t_{n}=T$ ) in double support, with $n-2$ intermediate configurations in single support and $T$ the duration of this single support. Consequently, the configurations will be defined by a small number of optimization parameters.

\subsection{Optimization parameters}

A parametric optimization problem has to be solved to design a cyclic bipedal gait with successive single supports and passive impacts (no impulsive torques are applied at impact). For a step defined on the time interval $[0, T]$ this problem depends on parameters to prescribe the $n-2$ intermediate configurations, the final velocity $\dot{\mathbf{q}}_{T}$ in the single support phase and, using the geometric model, the configuration of the biped at impact. Taking into account the conditions (26) and (27) the minimal number of parameters necessary to define the joint motion are: 
1. $(n-2) \times 12$ parameters are needed to define the $n-2$ intermediate configurations in single support phase.

2. The joint rates of the biped before the impact are also prescribed by twelve parameters, $\dot{\mathbf{q}}_{i}^{-}(i=1, \ldots, 12)$.

3. The position of the left foot denoted by $\left(y_{l f}, z_{l f}\right)$ in the horizontal plane as well as the situation of the middle of the hips defined by $\left(x_{h}, y_{h}, z_{h}, \theta_{h}\right)$ in double support phase are chosen as parameters.

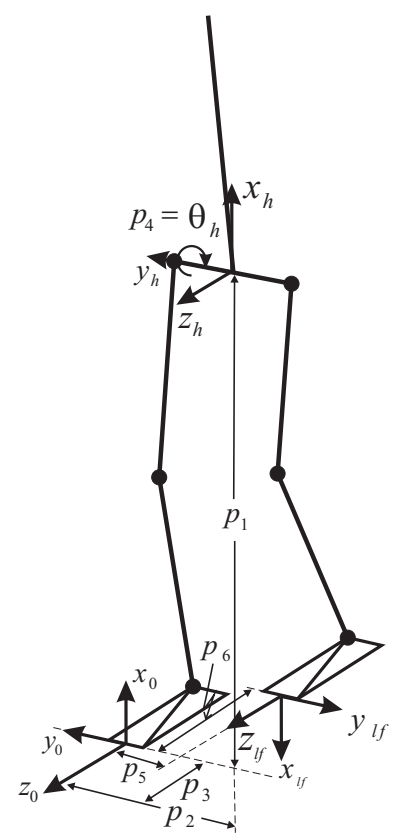

Fig. 2: The geometrie configuration of six parameters that define the initial configuration of the robot.

Then the total number of parameters is : $18+(n-2) \times 12$. Let us remark that to define the initial and final configurations for the step, when both leg feet touch the ground, nine parameters are required. However, we define these configurations with six parameters only.

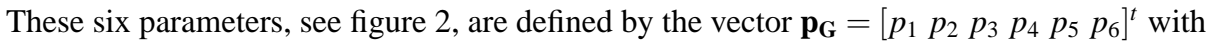
the following geometric configuration data:

1. $p_{1}:$ height of pelvis.

2. $p_{2}$ : position of the trunk following $y_{0}$ in the frame $R_{0}$.

3. $p_{3}$ : position of the trunk following $z_{0}$ in the frame $R_{0}$.

4. $p_{4}$ : orientation of the trunk in the sagittal plane.

5. $p_{5}:$ distance between the feet in the frontal plane.

6. $p_{6}:$ distance between the feet in the sagittal plane.

The others parameters, orientation of the middle of the hips in frontal and transverse planes, are fixed to zero. 
To summarize, the components of the joint evolution $\mathbf{q}$ are equal to the basis functions $\mathbf{q}_{i}$ (40) and we can write the joint motion with respect to the set of parameters $\mathbf{P}$ as

$$
\begin{aligned}
& \mathbf{q}=\varphi(\mathbf{P}, t) \\
& \dot{\mathbf{q}}=\dot{\varphi}(\mathbf{P}, t) \\
& \ddot{\mathbf{q}}=\ddot{\varphi}(\mathbf{P}, t)
\end{aligned}
$$

where $\varphi$ is the vector of components $\varphi_{i}(t)$ (40) defining the cubic splines for the joint $i$, $i=1, \ldots, 12$ the chosen vector of optimization parameters $\mathbf{P}$ can be written:

$$
\mathbf{P}=\left[\begin{array}{c}
\mathbf{P}_{1} \\
\mathbf{P}_{2} \\
\mathbf{P}_{3} \\
\mathbf{P}_{4}
\end{array}\right]=\left[\begin{array}{c}
\mathbf{q}_{\text {int } 1} \\
\mathbf{q}_{\text {int } 2} \\
\dot{\mathbf{q}}_{T} \\
\mathbf{p}_{\mathbf{G}}
\end{array}\right]
$$

Four our numerical tests $n=4$ and then two intermediate configurations $\mathbf{q}_{\text {int } 1}$ and $\mathbf{q}_{\text {int } 2}$ of the 3D biped in single support are considered.

\subsection{Criterion}

In the optimization process we consider, as cost functional $J$, the integral of the norm of the torque divided by the step length. In other words we are minimizing a quantity proportional to the energy lost in the actuators for a motion of one meter. This general form of minimal energy performance represents the losses by Joule effects for the electrical motors

$$
J=\frac{1}{d} \int_{0}^{T} \Gamma^{t} \Gamma d \mu
$$

To impose an average motion velocity, we take into account the equality constraint (39) in (46) as a penalty function, the cost functional $J$ can be write as

$$
J=\frac{1}{d} \int_{0}^{T} \Gamma^{t} \Gamma d \mu+\rho\left(V^{d} T-d\right)^{2}
$$

where $\rho>0$ is a penalty factor.

4.4 Statement of the optimization problem to design a cyclic walking gait for the 3D biped

Many values of parameters can give a periodic bipedal gait satisfying constraints (28)-(34). A parametric optimization process, that objective is to minimize $J$ under nonlinear constraints, is used to find a particular nominal motion with the splines (40) as basis functions. This optimization problem can be formally stated as

$$
\left.\begin{array}{l}
\text { Minimize } J(\mathbf{P}) \\
\text { subject to } g_{j}(\mathbf{P}) \leq 0 \quad j=1,2, \ldots, l
\end{array}\right\}
$$

where $J(\mathbf{P})$ is the cost functional to minimize with $l$ constraints $g_{j}(\mathbf{P}) \leq 0$ to satisfy. These constraints are given in section 3.2. The nonlinear constrained problem is solved by using the Matlab function fmincon. This optimization function provides an optimization algorithm based on the Sequential Quadratic Programming (SQP). There are forty-two parameters for 
this nonlinear optimization problem: twenty-four for the two intermediate configurations in single support, twelve for the joint rates before the impact and six to solve the inverse kinematics problem, subject to the constraints given by (28)-(34). The optimization problem (48) is numerically solved by using the exact analytic gradient of the cost functional with respect to the forty-two parameters. The calculation of this gradient is detailed in the following section.

\section{Gradient of the cost functional}

The optimization process uses the dynamic model (1) to calculate the torque vector $\Gamma$ for sampling times $\left\{0, \ldots, t_{k}, \ldots, T\right\}$ and to evaluate the cost functional (46) on the current step. Then $\Gamma$ is function of $\mathbf{q}, \dot{\mathbf{q}}$ and $\ddot{\mathbf{q}}$ of which the components equal basis functions $q_{i}$ (40) and their associated time derivatives $\dot{q}_{i}$ and $\ddot{q}_{i}, i=1, \ldots, 12$ :

$$
\Gamma=\Gamma(\mathbf{q}, \dot{\mathbf{q}}, \ddot{\mathbf{q}})
$$

The general formula of the gradient of the cost functional with respect to the vector $\mathbf{P}$ (45) is

$$
\begin{gathered}
\frac{\partial J}{\partial \mathbf{P}}=\frac{\partial}{\partial \mathbf{P}}\left(\frac{1}{p_{6}}\right) \int_{0}^{T} \Gamma^{t} \Gamma d \mu+\frac{1}{p_{6}} \frac{\partial}{\partial \mathbf{P}}\left(\int_{0}^{T} \Gamma^{t} \Gamma d \mu\right)+\rho \frac{\partial}{\partial \mathbf{P}}\left(\left(V^{d} T-p_{6}\right)^{2}\right) \\
=\left[\frac{\partial J}{\partial \mathbf{P}_{1}} \frac{\partial J^{t}}{\partial \mathbf{P}_{2}} \frac{\partial J^{t}}{\partial \mathbf{P}_{3}} \frac{\partial J^{t}}{\partial \mathbf{P}_{4}}\right]^{t}
\end{gathered}
$$

The calculation of each components of $\frac{\partial J}{\partial \mathbf{P}}$ will be detailed now.

- Calculation of $\frac{\partial J}{\partial \mathbf{P}_{1}}=\frac{\partial J}{\partial \mathbf{q}_{\text {int } 1}}$ and $\frac{\partial J}{\partial \mathbf{P}_{2}}=\frac{\partial J}{\partial \mathbf{q}_{\text {int } 2}}$ : The covered distance $p_{6}$ for a step does not depend on the intermediate configurations $\mathbf{q}_{\text {int } 1}$ and $\mathbf{q}_{\text {int } 2}$. Then the calculation of the gradient of the cost functional with respect to $\mathbf{P}_{1}$, the way being similar for $\mathbf{P}_{2}$, leads to

$$
\frac{\partial J}{\partial \mathbf{P}_{1}}=\frac{2}{p_{6}} \int_{0}^{T} \Gamma^{t} \frac{\partial \Gamma}{\partial \mathbf{q}_{\text {int } 1}} d \mu
$$

Tacking into account the relations (42), (43) and (44) and with the partial derivative formulas for composed functions, the partial derivative of $\Gamma$ with respect to $\mathbf{q}_{\text {int } 1}$ can be written,

$$
\frac{\partial \Gamma}{\partial \mathbf{q}_{i n t 1}}=\frac{\partial \Gamma}{\partial \mathbf{q}} \frac{\partial \mathbf{q}}{\partial \mathbf{q}_{i n t 1}}+\frac{\partial \Gamma}{\partial \dot{\mathbf{q}}} \frac{\partial \dot{\mathbf{q}}}{\partial \mathbf{q}_{\text {int } 1}}+\frac{\partial \Gamma}{\partial \ddot{\mathbf{q}}} \frac{\partial \ddot{\mathbf{q}}}{\partial \mathbf{q}_{\text {int } 1}}
$$

- Calculation of $\frac{\partial J}{\partial \mathbf{P}_{3}}=\frac{\partial J}{\partial \dot{\mathbf{q}}_{T}}$ : For the optimization problem the covered distance $d$ is not defined with the velocity vector $\dot{\mathbf{q}}_{T}$. Furthermore via the algebraic matrix equation (16) and (17) and the cyclic walking conditions (26) and (27), the initial velocity vector $\dot{\mathbf{q}}_{0}$ after impact is function of the final velocity vector before impact and the configuration of the 3D biped in double support such that:

$$
\dot{\mathbf{q}}_{0}=\dot{\mathbf{q}}_{0}\left(\mathbf{P}_{3}, \mathbf{P}_{4}\right)=\dot{\mathbf{q}}_{0}\left(\dot{\mathbf{q}}_{T}, \mathbf{p}_{\mathbf{G}}\right)
$$


In consequence using (42), (43), (44) and (53) the gradient of the cost functional with respect to $\mathbf{P}_{3}$ is:

$$
\frac{\partial J}{\partial \mathbf{P}_{3}}=\frac{2}{p_{6}} \int_{0}^{T} \Gamma^{t} \frac{\partial \Gamma}{\partial \dot{\mathbf{q}}_{T}} d \mu
$$

where,

$$
\frac{\partial \Gamma}{\partial \dot{\mathbf{q}}_{T}}=\frac{\partial \Gamma}{\partial \mathbf{q}} \frac{\partial \mathbf{q}}{\partial \dot{\mathbf{q}}_{T}}+\frac{\partial \Gamma}{\partial \dot{\mathbf{q}}} \frac{\partial \dot{\mathbf{q}}}{\partial \dot{\mathbf{q}}_{T}}+\frac{\partial \Gamma}{\partial \ddot{\mathbf{q}}} \frac{\partial \ddot{\mathbf{q}}}{\partial \dot{\mathbf{q}}_{T}}
$$

with,

$$
\begin{aligned}
\frac{\partial \mathbf{q}}{\partial \dot{\mathbf{q}}_{T}} & =\frac{\partial \varphi}{\partial \dot{\mathbf{q}}_{0}} \frac{\partial \dot{\mathbf{q}}_{0}}{\partial \dot{\mathbf{q}}_{T}}+\frac{\partial \varphi}{\partial \dot{\mathbf{q}}_{T}}, \\
\frac{\partial \dot{\mathbf{q}}}{\partial \dot{\mathbf{q}}_{T}} & =\frac{\partial \dot{\varphi}}{\partial \dot{\mathbf{q}}_{0}} \frac{\partial \dot{\mathbf{q}}_{0}}{\partial \dot{\mathbf{q}}_{T}}+\frac{\partial \dot{\varphi}}{\partial \dot{\mathbf{q}}_{T}}, \\
\frac{\partial \ddot{\mathbf{q}}}{\partial \dot{\mathbf{q}}_{T}} & =\frac{\partial \ddot{\varphi}}{\partial \dot{\mathbf{q}}_{0}} \frac{\partial \dot{\mathbf{q}}_{0}}{\partial \dot{\mathbf{q}}_{T}}+\frac{\partial \ddot{\varphi}}{\partial \dot{\mathbf{q}}_{T}} .
\end{aligned}
$$

- Calculation of $\frac{\partial J}{\partial \mathbf{P}_{4}}=\frac{\partial J}{\partial \mathbf{p}_{\mathbf{G}}}$ : The initial and final configurations $\mathbf{q}_{0}$ and $\mathbf{q}_{T}$ are found using the inverse geometric model thanks to the parameters vector $\mathbf{p}_{\mathbf{G}}=\left[p_{1}, p_{2}, p_{3}, p_{4}, p_{5}, p_{6}\right]^{t}$ and to the relation (26). The covered distance is directly function of $\mathbf{q}_{0}$ and $\mathbf{q}_{T}$. The calculation of the gradient of the cost functional with respect to $\mathbf{P}_{4}$ is given by

$$
\frac{\partial J}{\partial \mathbf{P}_{4}}=-\frac{1}{p_{6}^{2}} \frac{\partial p_{6}}{\partial \mathbf{p}_{\mathbf{G}}} \int_{0}^{T} \Gamma^{t} \Gamma d \mu+\frac{2}{p_{6}} \int_{0}^{T} \Gamma^{t} \frac{\partial \Gamma}{\partial \mathbf{p}_{\mathbf{G}}} d \mu+2 \rho\left(p_{6}-V^{d} T\right)
$$

where,

$$
\frac{\partial \Gamma}{\partial \mathbf{p}_{\mathbf{G}}}=\frac{\partial \Gamma}{\partial \mathbf{q}} \frac{\partial \mathbf{q}}{\partial \mathbf{p}_{\mathbf{G}}}+\frac{\partial \Gamma}{\partial \dot{\mathbf{q}}} \frac{\partial \dot{\mathbf{q}}}{\partial \mathbf{p}_{\mathbf{G}}}+\frac{\partial \Gamma}{\partial \ddot{\mathbf{q}}} \frac{\partial \ddot{\mathbf{q}}}{\partial \mathbf{p}_{\mathbf{G}}}
$$

Since the vector $\mathbf{p}_{\mathbf{G}}$ is function of the initial and final configurations $\mathbf{q}_{0}$ and $\mathbf{q}_{T}$ and using the relation (53) the partial derivative of $\mathbf{q}$ with respect of $\mathbf{p}_{\mathbf{G}}$ can be written

$$
\begin{gathered}
\frac{\partial \mathbf{q}}{\partial \mathbf{p}_{\mathbf{G}}}=\frac{\partial \varphi}{\partial \mathbf{q}_{0}} \frac{\partial \mathbf{q}_{0}}{\partial \mathbf{p}_{\mathbf{G}}}+\frac{\partial \varphi}{\partial \mathbf{q}_{T}} \frac{\partial \mathbf{q}_{T}}{\partial \mathbf{p}_{\mathbf{G}}}+\frac{\partial \varphi}{\partial \dot{\mathbf{q}}_{0}} \frac{\partial \dot{\mathbf{q}}_{0}}{\partial \mathbf{p}_{\mathbf{G}}}, \\
\frac{\partial \dot{\mathbf{q}}}{\partial \mathbf{p}_{\mathbf{G}}}=\frac{\partial \dot{\varphi}}{\partial \mathbf{q}_{0}} \frac{\partial \mathbf{q}_{0}}{\partial \mathbf{p}_{\mathbf{G}}}+\frac{\partial \dot{\varphi}}{\partial \mathbf{q}_{T}} \frac{\partial \mathbf{q}_{T}}{\partial \mathbf{p}_{\mathbf{G}}}+\frac{\partial \dot{\varphi}}{\partial \dot{\mathbf{q}}_{0}} \frac{\partial \dot{\mathbf{q}}_{0}}{\partial \mathbf{p}_{\mathbf{G}}}
\end{gathered}
$$

and

$$
\frac{\partial \ddot{\mathbf{q}}}{\partial \mathbf{p}_{\mathbf{G}}}=\frac{\partial \ddot{\varphi}}{\partial \mathbf{q}_{0}} \frac{\partial \mathbf{q}_{0}}{\partial \mathbf{p}_{\mathbf{G}}}+\frac{\partial \ddot{\varphi}}{\partial \mathbf{q}_{T}} \frac{\partial \mathbf{q}_{T}}{\partial \mathbf{p}_{\mathbf{G}}}+\frac{\partial \ddot{\varphi}}{\partial \dot{\mathbf{q}}_{0}} \frac{\partial \dot{\mathbf{q}}_{0}}{\partial \mathbf{p}_{\mathbf{G}}} .
$$

The partial derivative $\frac{\partial \dot{\mathbf{q}}_{0}}{\partial \mathbf{p}_{\mathbf{G}}}$, using the equation (27) with the constant matrix $\mathbf{E}$, can be rewritten such as

$$
\frac{\partial \dot{\mathbf{q}}_{0}}{\partial \mathbf{p}_{\mathbf{G}}}=\mathbf{E} \frac{\partial \dot{\mathbf{q}}^{+}}{\partial \mathbf{p}_{\mathbf{G}}} .
$$

The algebraic impact equations (16) and (17) can be concatenated such as:

$$
\left[\begin{array}{cc}
\mathbf{D} & \mathbf{D}_{12} \\
\mathbf{D}_{12}^{t} & \mathbf{0}_{6 \times 6}
\end{array}\right]\left[\begin{array}{l}
\mathbf{V}^{+} \\
\mathbf{I}_{13}
\end{array}\right]=\left[\begin{array}{l}
\mathbf{D V}^{-} \\
\mathbf{0}_{6 \times 1}
\end{array}\right]
$$


The partial derivative of the matrix equation (58) versus $\mathbf{p}_{\mathbf{G}}$ is:

$$
\left[\begin{array}{cc}
\frac{\partial \mathbf{D}}{\partial \mathbf{p}_{\mathbf{G}}} & \frac{\partial \mathbf{D}_{12}}{\partial \mathbf{p}_{\mathbf{G}}} \\
\frac{\partial \mathbf{D}_{12}^{t}}{\partial \mathbf{p}_{\mathbf{G}}} & \mathbf{0}_{6 \times 6}
\end{array}\right]\left[\begin{array}{c}
\mathbf{V}^{+} \\
\mathbf{I}_{13}
\end{array}\right]+\left[\begin{array}{cc}
\mathbf{D} & \mathbf{D}_{12} \\
\mathbf{D}_{12}^{t} & \mathbf{0}_{6 \times 6}
\end{array}\right]\left[\begin{array}{l}
\frac{\partial \mathbf{V}^{+}}{\partial \mathbf{p}_{\mathbf{G}}} \\
\frac{\partial \mathbf{I}_{13}}{\partial \mathbf{p}_{\mathbf{G}}}
\end{array}\right]=\left[\begin{array}{c}
\frac{\partial \mathbf{D} \mathbf{V}^{-}}{\partial \mathbf{p}_{\mathbf{G}}} \\
\mathbf{0}_{6 \times 1}
\end{array}\right]
$$

The matrix equation (59) can be rewritten

$$
\left[\begin{array}{l}
\frac{\partial \mathbf{V}^{+}}{\partial \mathbf{p}_{\mathbf{G}}} \\
\frac{\partial \mathbf{I}_{13}}{\partial \mathbf{p}_{\mathbf{G}}}
\end{array}\right]=W\left(-\left[\begin{array}{cc}
\frac{\partial \mathbf{D}}{\partial \mathbf{p}_{\mathbf{G}}} & \frac{\partial \mathbf{D}_{12}}{\partial \mathbf{p}_{\mathbf{G}}} \\
\frac{\partial \mathbf{D}_{12}^{t}}{\partial \mathbf{p}_{\mathbf{G}}} & \mathbf{0}_{6 \times 6}
\end{array}\right]\left[\begin{array}{c}
\mathbf{V}^{+} \\
\mathbf{I}_{13}
\end{array}\right]+\left[\begin{array}{c}
\frac{\partial \mathbf{D} \mathbf{V}^{-}}{\partial \mathbf{p}_{\mathbf{G}}} \\
\mathbf{0}_{6 \times 1}
\end{array}\right]\right)
$$

with

$$
W=\left[\begin{array}{cc}
\mathbf{D} & \mathbf{D}_{12} \\
\mathbf{D}_{12}^{t} & \mathbf{0}_{6 \times 6}
\end{array}\right]^{-1}
$$

and

$$
\begin{aligned}
& \mathbf{W}_{11}=\mathbf{D}^{-1}\left(\mathbf{I}_{18 \times 18}-\mathbf{D}_{12}\left(\mathbf{D}_{12}^{t} \mathbf{D}^{-1} \mathbf{D}_{12}\right)^{-1} \mathbf{D}_{12}^{t} \mathbf{D}^{-1}\right) \\
& \mathbf{W}_{21}=\left(\mathbf{D}_{12}^{t} \mathbf{D}^{-1} \mathbf{D}_{12}\right)^{-1} \mathbf{D}_{12}^{t} \mathbf{D}^{-1}=\mathbf{W}_{12}^{t} \\
& \mathbf{W}_{22}=-\left(\mathbf{D}_{12}^{t} \mathbf{D}^{-1} \mathbf{D}_{12}\right)^{-1} .
\end{aligned}
$$

Finally we have:

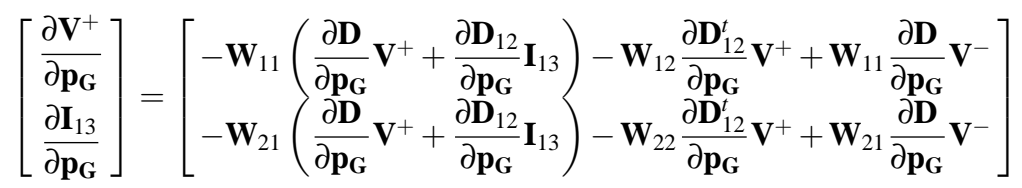

With the knowledge of $\mathbf{q}_{T}$, the solutions $\mathbf{V}^{+}$and $\mathbf{I}_{13}$ of the impact equation (58) and using (18) which stipulates that before impact the stance foot is motionless such as $\mathbf{V}^{-}=\left[\mathbf{0}_{1 \times 3}, \mathbf{0}_{1 \times 3}, \dot{\mathbf{q}}^{-T}\right]^{t}$ where $\dot{\mathbf{q}}^{-}=\dot{\mathbf{q}}_{T}$, variable $\frac{\partial \mathbf{q}^{+}}{\partial \mathbf{p}_{\mathbf{G}}}$ is equal to:

$$
\frac{\partial \mathbf{q}^{+}}{\partial \mathbf{p}_{\mathbf{G}}}=\left(-\mathbf{W}_{11}\left(\frac{\partial \mathbf{D}}{\partial \mathbf{p}_{\mathbf{G}}}\left(\mathbf{V}^{+}-\mathbf{V}^{-}\right)+\frac{\partial \mathbf{D}_{12}}{\partial \mathbf{p}_{\mathbf{G}}} \mathbf{I}_{13}\right)-\mathbf{W}_{12} \frac{\partial \mathbf{D}_{12}^{t}}{\partial \mathbf{p}_{\mathbf{G}}} \mathbf{V}^{+}\right)_{((7: 18) \times 1)}
$$

To summarize, in this section 5 we present the main necessary connections to calculate the gradient of the cost functional. Of course the computation of this gradient is heavy. However, we can remark that only the terms $\frac{\partial \Gamma}{\partial \mathbf{q}}, \frac{\partial \Gamma}{\partial \dot{\mathbf{q}}}$ and $\frac{\partial \Gamma}{\partial \ddot{\mathbf{q}}}$ have to be included in the recursive dynamics computation defined by the Newton-Euler equations. Their calculations are detailed in [35].

In conclusion the algorithm to obtain an optimal cyclic walking gait for the biped can be summarized by:

Step 1: Give initial values for each components of the parameter vector $\mathbf{P}$ (45).

Step 2: With the parameters $\mathbf{P}_{4}=\mathbf{p}_{\mathbf{G}}$ compute the initial configuration and from the equation (26) the final configuration.

Step 3: With the final configurations, the parameters $\mathbf{P}_{3}=\dot{\mathbf{q}}_{T}$ and the equations (16), (17) and (27) compute the initial velocity $\dot{\mathbf{q}}_{0}$. 
Step 4: For time $t_{1}=0$ to $t_{n}=T$, compute the spline functions (40) for the initial and final configurations and the parameters $\mathbf{P}_{1}=\mathbf{q}_{\text {int } 1}$ and $\mathbf{P}_{2}=\mathbf{q}_{\text {int } 2}$. Compute their first and second derives with respect to time.

Step 5: For sampling time $\left\{0, \ldots, t_{k}, \ldots, T\right\}$, solve recursively the inverse dynamics (2)-(11) to compute the torques, the position of the Center of Pressure $\mathrm{CoP}$, the constraints and the partial derivatives $\frac{\partial \Gamma}{\partial \mathbf{q}}, \frac{\partial \Gamma}{\partial \dot{\mathbf{q}}}$ and $\frac{\partial \Gamma}{\partial \ddot{\mathbf{q}}}$.

Step 6: Using the Euler method approximate the integral of the square vector of torques to compute the cost functional and its gradient respect to the parameter vector $\mathbf{P}$.

Step 7: If the condition to stop the optimization are satisfied, stop, in other case go to step 1 with a new parameter vector given by the optimization process.

\section{Simulations results}

To validate our proposed method, we present the results of an optimal motion for the biped shown in figure 1 . The desired trajectory was obtained by the optimization process presented in Section IV with a desired average velocity of $1 \mathrm{~m} / \mathrm{s}$. The case of optimisation with both finite difference and analytical gradients of the cost functional are considered. In the case of finite difference gradient, the algorithm was terminated after 513 iterations (22858 function evaluations). The final value of the objective function was 9354.792 and the total elapsed time was $3355.92 \mathrm{~s}$. For the analytical gradient, the algorithm was terminated after 407 iterations (18236 function evaluations), with a total computation time of $6822.48 \mathrm{~s}$ and the final value of the objective function 6295.95. The introduction of the analytical gradient, showed a better stability in the optimization process and a good convergence to find a minimum local.

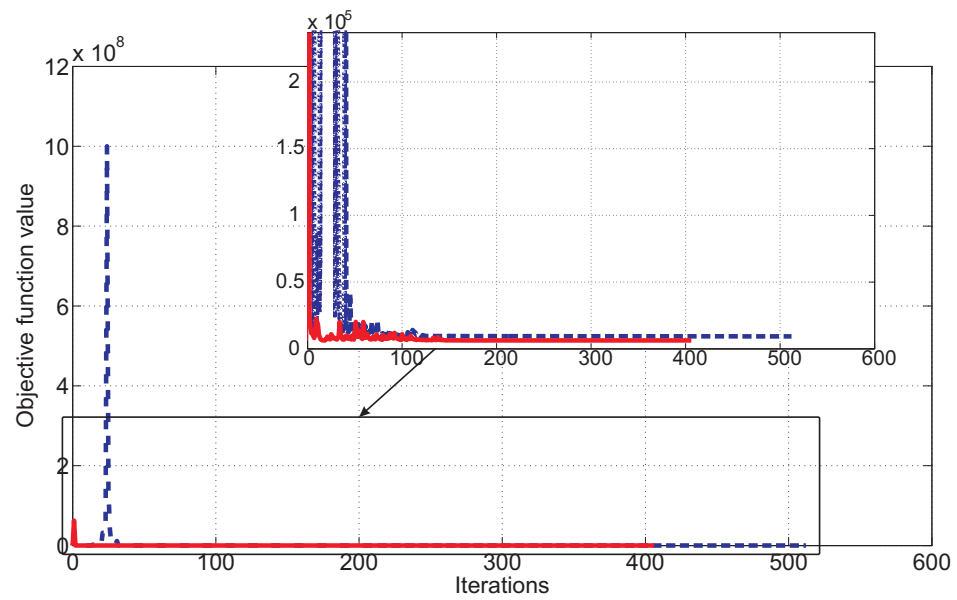

Fig. 3: Comparison of optimization processes: curves corresponding to the convergence behavior of the algorithm with the analytical and finite difference gradients are solid and dash-dotted, respectively.

The convergence speed and the number of iterations are compared for the optimization process with the analytical gradient and finite difference gradients of the cost functional. 
Figure 3 shows the number of iterations for both optimization processes, while table 3 shows a summary of the results for several walking rates.

\begin{tabular}{ccc}
\hline Physical Parameters & Mass $(k g)$ & Length $(m)$ \\
& & \\
\hline Torso & 40.55 & $d_{7}=0.120$ \\
Hip joints & 2.04 & linked to torso \\
Thigh & 2.08 & $d_{4}=0.3$ \\
Shin & 1.75 & $d_{3}=0.3$ \\
Ankle joints & 0.65 & $d_{1}=0.105$ \\
Foot & 1.64 & $L_{p}=0.214, l_{p}=0.136$ \\
\hline
\end{tabular}

Table 2: Parameters of the 3D biped, see figure 1

Figure 4 shows the evolution of the optimal motion for one step with duration, of a single support, equal $0.4 \mathrm{~s}$. For the simulation, we use the physical parameters given in table 2. The bipedal robot has the height of $1.30 \mathrm{~m}$ and the weight of $56.86 \mathrm{~kg}$. The inertia of each link are also taken into account in the dynamic model.

The results shown have been obtained with $T_{s}=0.4 \mathrm{~s}$. The optimal motion is such that the step length is $0.4 \mathrm{~m}$ and the walking rate is imposed to $1 \mathrm{~m} / \mathrm{s}$. The simulation of the optimal motion for one step is illustrated in figure 4 and for 3 walking steps in figure 9 .

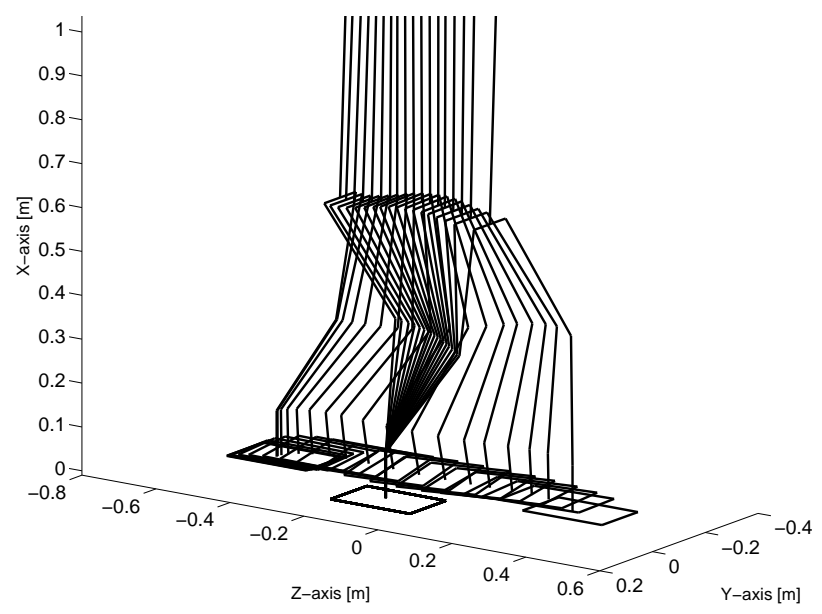

Fig. 4: Stick animation of a simulation of walking biped taking one step.

The normal components of the ground reaction forces of the stance foot during one step are presented in figure 5. The average vertical reaction force is $547.81 \mathrm{~N}$, which is coherent with the weight of the robot which the mass equals $56.86 \mathrm{Kg}$. In the optimisation process, the chosen friction coefficient is 0.7 -, the condition of non sliding is satisfied as it can be seen in figure 5 .

The figure 6 shows the evolution of the trajectory of the center of pressure $\mathrm{CoP}$ considering one and two intermediate configurations. In both cases, the evolution of the $C o P$ is 


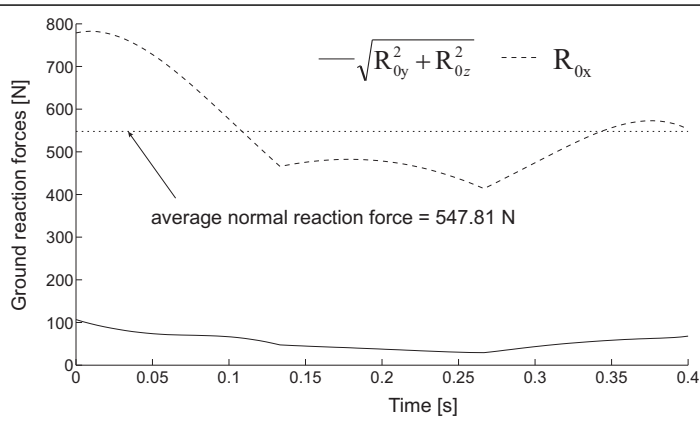

Fig. 5: The ground reaction forces during the single support phase.

always inside the rectangle determined by $l_{p}=0.136 \mathrm{~m}$ and $L_{p}=0.214 \mathrm{~m}$, that is, the robot maintains the balance during the motion. From figure 6, it can be seen that considering two intermediate configurations, the evolution of the $\mathrm{CoP}$ presents amplitudes lower. Because the minimal distance between of $\mathrm{CoP}$ and the boundary of the foot is large, smaller foot is acceptable for this cyclic motion. The criterion cost, considering only one intermediate configuration, is $6472.5 \mathrm{~N}^{2} \cdot \mathrm{m} \cdot \mathrm{s}$.

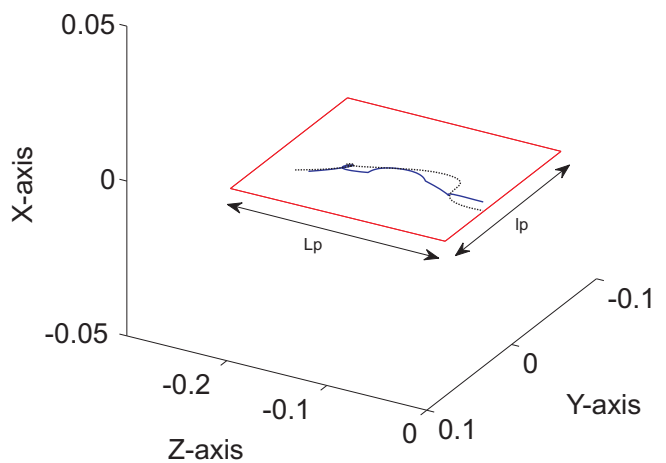

Fig. 6: The evolution of $\mathrm{CoP}$ trajectory: curves corresponding to $\mathrm{CoP}$ trajectory considering one and two intermediate configurations are dotted and solid, respectively.

In figure 7, the evolution of the cost criteria is drawn as function of several walking rates. A faster walking motion than $1.2 \mathrm{~m} / \mathrm{s}$ can not be obtained.

The curves in figure 8, illustrate the evolution of torques cost of each joint of stance and swing leg as function of the walking rate. The torques cost of swing leg is less important than the torques cost of stance leg. For slow motion, less than $0.6 \mathrm{~m} / \mathrm{s}$, the torque cost of the stance hip is less important, while that for a walking gait faster than $0.9 \mathrm{~m} / \mathrm{s}$ this torque cost increases considerably. 


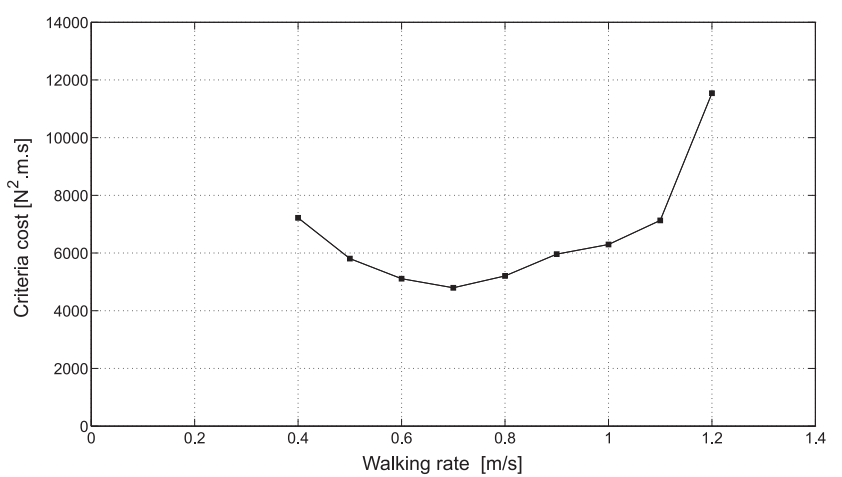

Fig. 7: The evolution of the cost criteria with analytical gradient.

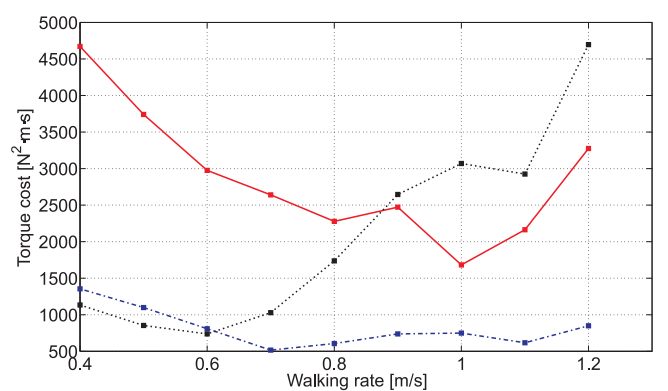

(a) stance leg

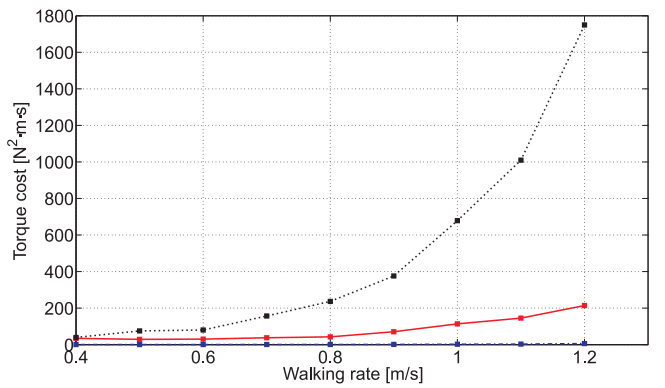

(b) swing leg

Fig. 8: The cost of the joint torques: curves corresponding to the torque costs of the ankle and knee are dash-dotted and solid, respectively. The dotted curve corresponds to the torque cost of the hip.

\section{Conclusion}

Optimal joint reference trajectories for cyclic walking gaits of a 3D biped have been found. A methodology to design such optimal trajectories is developed. The definition of optimal trajectories is useful to test a robot design. In order to use a classical optimization technique, the optimal trajectory is described by a set of parameters: we choose to define the evolution of the actuated relative angle as spline functions. A cyclic solution is desired. The number 


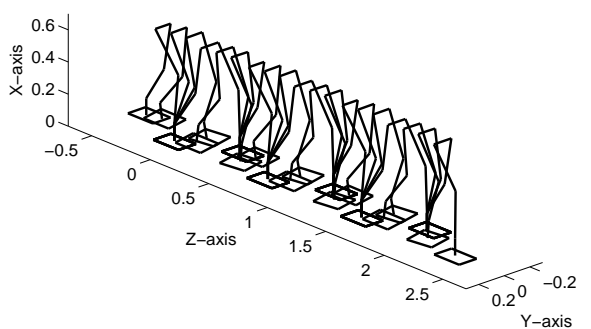

Fig. 9: Cyclic motion of the bipedal robot.

\begin{tabular}{|c|c|c|c|c|c|}
\hline Walking rate $(\mathrm{m} / \mathrm{s})$ & Gradient & Number of iterations & Function evaluations & Optimum value & Time (sec) \\
\hline \multirow{2}{*}{0.4} & analytical & 593 & 26461 & 7221.53 & 10677.15 \\
\hline & finite differences & 487 & 21235 & 7218.6 & 9071.95 \\
\hline \multirow{2}{*}{0.5} & analytical & 398 & 17780 & $\mathbf{5 8 0 7 . 5 7}$ & 6862.54 \\
\hline & finite differences & 401 & 17499 & 5809.45 & 7567.15 \\
\hline \multirow{2}{*}{0.6} & analytical & 560 & 24964 & 5108.99 & 9488.86 \\
\hline & finite differences & 488 & 21260 & 4998.15 & 9280.12 \\
\hline \multirow{2}{*}{0.7} & analytical & 449 & 20145 & 4794.33 & 7677.10 \\
\hline & finite differences & 772 & 33590 & 4815.49 & 17895.15 \\
\hline \multirow[b]{2}{*}{0.8} & analytical & 564 & 25212 & 5205.01 & 9689.82 \\
\hline & finite differences & 813 & 35356 & 6104.05 & 14738.13 \\
\hline \multirow{2}{*}{0.9} & analytical & 438 & 19590 & 5961.33 & 7471.96 \\
\hline & finite differences & 895 & 39767 & 5984.20 & 5820.21 \\
\hline \multirow{2}{*}{1.0} & analytical & 407 & 18236 & 6295.95 & 6822.48 \\
\hline & finite differences & 513 & 22858 & 9354.79 & 3355.92 \\
\hline \multirow{2}{*}{1.1} & analytical & 452 & 20208 & 7130.79 & 7856.68 \\
\hline & finite differences & 429 & 18613 & 15856 & 8321.76 \\
\hline
\end{tabular}

Table 3: Optimization results. For each optimal motion, the vector of initial parameters was the same for both optimal process. All of the simulations were performed on computer equipped with a processor $2.0 \mathrm{GHz}$ Core Duo from Intel.

of the optimization variables is reduced by taking into account of the cyclicity condition explicitly.

Some inequality constraints such as the limits on the torques and the velocities, the condition of no sliding during motion and impact, some limits on the motion of the free leg are taken into account. The cost functional is calculated from the integral of the torques norm. The torques are computed for sampling times using the inverse dynamic model. This 
model is obtained with the recursive Newton-Euler algorithm. The reference frame is the stance foot.

In the optimization process, a calculation of the gradient by a finite approximation can generate numerical errors for the Hessian computation. Then to improve the convergence of the optimization algorithm, the explicit analytical gradient with respect to the optimization parameters is calculated using the recursive equations of the dynamic model. Optimal motions for a given duration of the step and for a walking rate imposed have been obtained. The numerical results obtained are realistic with respect to the size of the walker under study.

The proposed method to define an optimal motion will be tested, considering a sub-phase of rotation of the supporting phase about the toe, closer to human. Another perspective is to evaluate the gradient of constraints with respect to the optimization parameters.

\section{References}

1. A. A. Grishin, A. M. Formal'sky, A. V. Lensky, and S. V. Zhitomirsky, "Dynamic walking of a vehicle with two telescopic legs controlled by two drives," Int. J. of Robotics Research, vol. 13, no. 2, pp. 137147,1994

2. M. Rostami and G. Besonnet, "Impactless sagital gait of a biped robot during the single support phase," in Proceedings of International Conference on Robotics and Automation, 1998, pp. 1385-1391.

3. A. E. Bryson and Y. C. Ho, Applied optimal control. Wiley, New-York, 1995.

4. Y. C. Chen, "Solving robot trajectory planning problems with uniform cubic b-splines," Optimal Control Applications and Methods, vol. 12, no. 4, pp. 247-262, 1991.

5. A. D. Luca, L. Lanari, and G. Oriolo, "A sensitive approach to optimal spline robot trajectories," Automatica, vol. 27, no. 3, pp. 535-539, 1991.

6. J. P. Ostrowski, J. P. Dessai, and V. Kumar, "Optimal gait selection for nonholonomic locomotion systems," International Jounal of Robotics Research, vol. 19, no. 3, pp. 225-237, 2000

7. A. Dürrbaum, W. Klier, and H. Hahn, "Comparaison of automatic and symbolic differentiation in mathematical modeling and computer simulation of rigid-body," Multibody System Dynamics, vol. 7, no. 4, pp. 331-355, 2002.

8. S. H. Lee, J. Kim, F. C. Park, M. Kim, and J. E. Bobrow, "Newton-type algorithm for dynamics-based robot movement optimization," IEEE Transactions on Robotics and Automation, vol. 21, no. 4, pp. 657667,2005

9. S. Miossec and Y. Aoustin, Dynamical synthesis of a walking cyclic gait for a biped with point feet. Special issue of lecture Notes in Control and information Sciences, Fast Motions in Biomechanics and Robotics, Ed. M. Dhiel and K. Mombaur, Springer-Verlag, 2006.

10. J. E. Bobrow, F. C. Park, and A. Sideris, Recent advance on the algorithme optimization of robot motion. Special issue of lecture Notes in Control and information Sciences, Fast Motions in Biomechanics and Robotics, Ed. M. Dhiel and K. Mombaur, Springer-Verlag, 2006.

11. L. Roussel, C. C. de Wit, and A. Goswami, "Generation of energy optimal complete gait cycles for biped," in Proc. of the IEEE Conf. on Robotics and Automation, 2003, pp. 2036-2042.

12. V. V. Beletskii and P. S. Chudinov, "Parametric optimization in the problem of bipedal locomotion," Izv. An SSSR. Mekhanika Tverdogo Tela [Mechanics of Solids], no. 1, pp. 25-35, 1977.

13. G. Bessonnet, S. Chesse, and P. Sardin, "Generating optimal gait of a human-sized biped robot," in Proc. of the fifth International Conference on Climbing and Walking Robots, 2002, pp. 717-724.

14. P. H. Channon, S. H. Hopkins, and D. T. Pham, "Derivation of optimal walking motions for a bipedal walking robot," Robotica, vol. 2, no. 165-172, 1992.

15. F. Zonfrilli, M. Oriolo, and T. Nardi, "A biped locomotion strategy for the quadruped robot sony ers-210," in Proc. of the IEEE Conf. on Robotics and Automation, 2002, pp. 2768-2774.

16. C. Chevallereau. and Y. Aoustin, "Optimal reference trajectories for walking and running of a biped," Robotica, vol. 19, no. 5, pp. 557-569, 2001.

17. B. Martin and J. E. Bobrow, "Minimum effort motions for open chained manipulators with taskdependent end-effort constraints," International Journal of Robotics Research, vol. 18, no. 2, pp. 213$224,1999$.

18. J. Lo, G. Huang, and D. Metaxas, "Human motion planning based on recursive dynamics and optimal control techniques," Multibody systems dynamics, vol. 8, pp. 433-458, 2002.

19. T. Saidouni and G. Bessonnet, "Generating globally optimised saggital gait cycles of a biped robot," Robotica, vol. 21, no. 2, pp. 199-210, 2003. 
20. L. Hu, C. Zhou, and Z. Sun, "Biped gait optimization using spline function based probability model," in Proc. of the IEEE Conference on Robotics and Automation, pp. 830-835, 2006.

21. J. Marot, "Contribution à synthèse dynamique optimale de la marche," Ph.D. dissertation, Université de Poitiers France, 2007.

22. J. E. Bobrow, B. Martin, G. Sohl, E. C. Wang, F. C. Park, and K. Kim, "Optimal robot motions for physical criteria," Journal of Robotic Systems, vol. 18, no. 12, pp. 785-792, 2001.

23. D. P. Garg and M. Kumar, "Optimization techniques applied to multiple manipulators for path panning and torque minimization," Engineering Applications of Intelligence, vol. 15, pp. 241-252, 2002.

24. J. Angeles, Fundamentals of Robotic Mechanical Systems. Springer-Verlag, New York, 1997.

25. W. Khalil and J. Kleinfinger, "A new geometric notation for open and closed loop robots," Proc. of the IEEE Conference on Robotics and Automation, pp. 1174-1180, 1985.

26. W. Khalil and E. Dombre, Modeling, identification and control of robots. Hermes Sciences Europe, 2002.

27. J. Y. S. Luh, M. W. Walker, and R. C. P. Paul, "Resolved-acceleration control of mechanical manipulators," IEEE Trans. on Automatic Control, vol. 25, no. 3, pp. 468-474, 1980.

28. A. Formal'sky, Locomotion of Anthropomorphic Mechanisms. Nauka, Moscow [In Russian], 1982.

29. M. Sakaguchi, A. S. J. Furushu, and E. Koizumi, "A realization of bounce gait in a quadruped robot with articular-joint-type legs," Proc. of the IEEE Conference on Robotics and Automation, pp. 697-702, 1995.

30. M.W.Walker and D.E.Orin, "Efficient dynamic computer simulation of robotics mechanism," Trans. of ASME, J. of Dynamic Systems, Measurement and Control, vol. 104, pp. 205-211, 1982.

31. M. Vukobratovic and B. Borovac, "Zero moment point-thirty five years of its life," Int. Journal of Humanoid Robotics, vol. 1, no. 1, pp. 157-173, 2004.

32. M. Vukobratovic and Y. Stepanenko, "On the stability of anthropomorphic systems," Mathematical Biosciences, vol. 15, pp. 1-37, 1972.

33. M. Vukobratovic and B. Borovac, Biped Locomotion-Dynamics, Stability, Control and Application. Springer-Verlag, 1990.

34. N. H. Ahlberg and et al., The theory of splines and their applications. New York: Academic, 1967.

35. D. Tlalolini Romero, "Génération de mouvements optimaux de marche pour des robots bipèdes $3 \mathrm{~d}$," $\mathrm{PhD}$ thesis, Ecole Centrale de Nantes, Université de Nantes, Nantes, France, Decembre 2008. 